\title{
Chitosan/DNA Polyelectrolyte Complex Membranes for Controlling Cell Spreading and Aggregation
}

\author{
Naoki Kawazoe ${ }^{1, *}$, Yujiro Narita ${ }^{2}$, Guoping Chen ${ }^{1}$, Tadaatsu Satomi ${ }^{2}$ and Tetsuya Tateishi ${ }^{1,2}$ \\ ${ }^{1}$ Biomaterials Center, National Institute for Materials Science, Namiki 1-1, Tsukuba, Ibaraki 305-0044, Japan and \\ ${ }^{2}$ Graduate School of Science and Engineering, Tokyo Denki University, Ishizaka, Hatoyama-machi, Hiki-gun, Saitama \\ 350-0394, Japan
}

\begin{abstract}
Three kinds of polyelectrolyte complex membranes were prepared by mixing chitosan and DNA at the ratios of $3: 1,1: 1$, and 1:3. The effects of the membranes on cell adhesion, spreading, and viability were investigated. The membrane with the high ratio of chitosan had a less hydrophilic surface. The surface of the polyelectrolyte complex membranes became rough as the DNA content increased. The ratio of DNA to chitosan showed some effect on cell adhesion, spreading, and viability. Human mesenchymal stem cells adhered and spread on membranes prepared at chitosan/DNA ratios of 1:1 and 3:1, while they did not on membranes prepared at a chitosan/DNA ratio of 1:3. Cells aggregated on the membrane prepared at a chitosan/DNA ratio of 1:3. Cell viability was also higher on membranes prepared at chitosan/DNA ratios of $1: 1$ and 3:1 than that on the membrane prepared at a chitosan/DNA ratio of 1:3. The membrane with a high content of chitosan facilitated cell adhesion and spreading, while a high content of DNA suppressed cell adhesion and spreading.
\end{abstract}

\section{INTRODUCTION}

Chitosan is a natural cationic polysaccharide that has been widely used for biomedical applications because of its biocompatibility and biodegradability [1-4]. It has been used as a non-viral vector for gene delivery to cells [5-7] because it can form a polyelectrolyte complex with DNA [8]. The formation of the complex is associated with neutralization of the negative charge of DNA by the positive charge of chitosan. As a result, the DNA molecules become more compact and permeate the cell membrane more easily.

In recent years, the chitosan/DNA complex has also been used to prepare porous scaffolds for tissue engineering [9, 10] reported the preparation of porous chitosan/DNA scaffolds. They demonstrated that the porosity of chitosan/DNA complexes and the intercalation and groove-binding rate of Daunorubicin hydrochloride in the complexes could be controlled by rinsing and re-rinsing with PBS of different $\mathrm{pH}$ values. The porous chitosan/DNA scaffolds showed nontoxic effects on osteoblast-like cells and caused only a mild tissue response when implanted in the backs of rats.

Besides the complex structure, the charge of the chitosan/DNA-derived material can also be easily controlled by changing the ratio of chitosan and DNA. The charge can vary from positive to neutral to negative by adjusting the two components. Surface properties such as charge, wettability, roughness, and hardness have been reported to affect cell functions such as attachment, spreading, migration, proliferation, and differentiation [11-16]. However, the effects of the composition in chitosan/DNA polyelectrolyte complexes on cell spreading and aggregation are still unclear.

*Address correspondence to this author at the Biomaterials Center, National Institute for Materials Science, Namiki 1-1, Tsukuba, Ibaraki 305-0044, Japan; E-mail: KAWAZOE.Naoki@nims.go.jp
In the present study, we prepared a few kinds of chitosan/DNA complex membranes with different ratios of chitosan and DNA, and examined the effects of the monomer ratios of chitosan/DNA on cell functions including cell spreading and aggregation.

\section{MATERIALS AND METHODS}

\section{Preparation of Chitosan/DNA Complex Membranes}

Five hundred milligrams of fully deacetylated chitosan (average molecular weight of $1.3 \times 10^{5} \mathrm{Da}$ ) was dissolved in $40 \mathrm{~mL}$ of $0.2 \mathrm{M} \mathrm{HCl}$ aqueous solution. Next, $0.2 \mathrm{M} \mathrm{NaOH}$ aqueous solution was added to the chitosan solution to adjust the $\mathrm{pH}$ to 5.0 and the total volume of the solution was adjusted to $100 \mathrm{~mL}$ with Milli-Q water. DNA (average molecular weight of $1.7 \times 10^{5} \mathrm{Da}$ ) extracted from salmon testes was dissolved in $100 \mathrm{~mL}$ of Milli-Q water. The DNA solution was dropwisely added into the chitosan solution under stirring. Three complexes were prepared with the monomer ratios of chitosan: DNA of $1: 3,1: 1$, and $3: 1$. The mixtures were further stirred at room temperature for another $1 \mathrm{~h}$. The resulting insoluble complex materials were collected by centrifugation at $780 \mathrm{~g}$ for $5 \mathrm{~min}$ and the precipitates were alternately washed with Milli-Q water and $0.2 \mathrm{~N} \mathrm{HCl}$. This washing was repeated three times. After being washed, the precipitates were resuspended in Milli-Q water to prepare suspension solutions of $2 \mathrm{mg} / \mathrm{mL}$. The suspension solutions $(2250 \mu \mathrm{L})$ were dropped onto a square polystyrene (PS) plate $(1.5 \mathrm{~cm} \times 1.5 \mathrm{~cm})$ cut from a cell culture plate (Becton Dickinson Labware, Franklin Lakes, NJ, USA), air-dried for 48 h, and then dried in vacuum for another $4 \mathrm{~h}$. The chitosan/DNA complex membranes were formed on the PS substrates. The surface structure of membranes was observed with SEM (JSM-5600LV, JEOL, Tokyo, Japan). 


\section{Contact Angle Measurement}

The contact angles of water on the chitosan/DNA polyelectrolyte complex membranes were measured with a sessile method using an automatic contact angle meter (CA-W, Kyowa Interface Science Co., Ltd., Saitama, Japan). The measurements were made on dry membranes at room temperature. At least five replications were carried out for each membrane.

\section{Cell Seeding and Culture}

Human bone marrow-derived mesenchymal stem cells (hMSC) were obtained from Osiris (Worthington Biochemical, Lakewood, NJ, USA) at passage 2. The cells were seeded in T-75 culture flasks using the proliferation medium from Osiris. The proliferation medium contained $440 \mathrm{~mL}$ hMSC basal medium, $50 \mathrm{~mL}$ mesenchymal cell growth supplement, $10 \mathrm{~mL} 200 \mathrm{mM} \mathrm{L}$-glutamine, and $0.5 \mathrm{~mL}$ penicillin/streptomycin mixture. The cells were further subcultured once after reaching confluence and used at passage 4 . The cells were collected by treatment with trypsin/EDTA solution, and suspended in proliferation medium at a density of $1.0 \times 10^{6}$ cells $/ \mathrm{mL}$. The polyelectrolyte complex membranes on the PS substrates were sterilized with $70 \%$ (v/v) ethanol, washed twice with sterilized Milli-Q water, placed in the wells of a 12-well plate containing $1.0 \mathrm{~mL}$ proliferation medium, and covered with glass rings having an inner diameter of $1.0 \mathrm{~cm}$ to protect against cell leakage during cell seeding. The hMSC suspension solution $\left(1 \times 10^{6}\right.$ cells $/ \mathrm{mL}, 1 \mathrm{~mL} /$ membrane) was dropped onto the polyelectrolyte complex membrane and cultured under an atmosphere of $5 \% \mathrm{CO}_{2}$ at $37^{\circ} \mathrm{C}$ for $3 \mathrm{~h}$. After that, the samples were removed for SEM observation. The cells were fixed with $1.0 \%$ glutaraldehyde solution in PBS at room temperature for $2 \mathrm{~h}$. After being rinsed three times with water, the samples were freeze-dried for $12 \mathrm{~h}$. The samples were then sputter coated with platinum using an ion coater (ESC-101, ELINOX, Tokyo, Japan) and observed with SEM.

\section{Cell Viability Assay}

Cell viability was measured with a colorimetric assay using Cell Proliferation Reagent WST-1 (Roche Diagnostics, Mannheim, Germany). The polyelectrolyte complex suspension solutions $(2 \mathrm{mg} / \mathrm{mL}, 283 \mu \mathrm{L})$ were dropped onto a $96-$ well PS cell culture plate (Becton Dickinson Labware, Franklin Lakes, NJ, USA), air-dried for $48 \mathrm{~h}$, and then dried in vacuum for another $4 \mathrm{~h}$. The plates were then seeded with $100 \mu \mathrm{L}$ hMSCs $\left(1 \times 10^{6}\right.$ cells $\left./ \mathrm{mL}\right)$ and cultured at $37^{\circ} \mathrm{C}$ for 3 h. After culture, $10 \mu \mathrm{L}$ of WST-1 reagent solution was added per well, and the mixture was incubated at $37^{\circ} \mathrm{C}$ for $2 \mathrm{~h}$. Then, $0.1 \mathrm{~mL}$ solution from each well was transferred to a new 96-well plate and absorbance at $440 \mathrm{~nm}$ was measured with a microtiter plate reader (Benchmark, Bio-Rad Laboratories, Hercules, California, USA). Blank wells containing only cell culture medium and WST-1 solution were also prepared, incubated, and read. The blank value was subtracted from each of the experimental values as background.

\section{RESULTS}

\section{Formation of Chitosan/DNA Complex Membranes}

Chitosan/DNA polyelectrolyte complexes were prepared by dropping DNA aqueous solution into chitosan aqueous solution. The chitosan/DNA polyelectrolyte complexes were resuspended and used for membrane preparation. The suspension solution was dropped onto polystyrene plates, either treated or untreated with cell culture. Polyelectrolyte complex membranes were formed on the treated polystyrene plates, but the membranes on the untreated plates were broken during drying. The surface property of the PS substrate was found to affect the formation of the polyelectrolyte complex membrane. The hydrophilic property of the treated polystyrene plate might contribute the stabilization of the membrane via hydrogen bonding. SEM observation showed that the surface of the polyelectrolyte complex membranes became rough with increased DNA content, as shown in Fig. (1).

The water contact angles on the membranes are listed in Table 1. The water contact angle on the chitosan/DNA membrane prepared at a chitosan/DNA ratio of 3:1 was higher than those at chitosan/DNA ratios of $1: 3$ and 1:1. This might be caused by the hydrophobic property of chitosan ratios of $1: 3$ and $1: 1$.

a

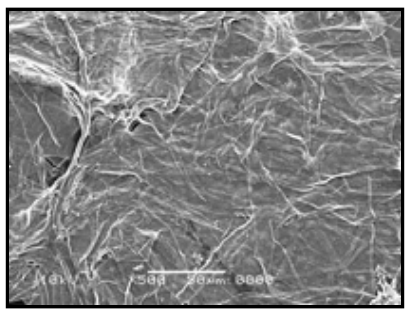

b

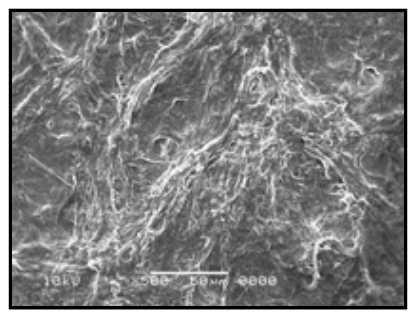

C

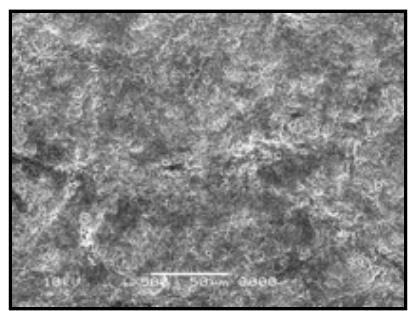

Fig. (1). SEM photographs of the chitosan/DNA polyelectrolyte complex membranes with the chitosan: DNA monomer ratios of (a) $1: 3$, (b) $1: 1$, and (c) $3: 1$.

Table 1. Water Contact Angles on the Chitosan/DNA Polyelectrolyte Complex Membranes with Different Ratios of Chitosan and DNA

\begin{tabular}{|c|c|c|c|}
\hline Chitosan:DNA (monomer) & $1: 3$ & $1: 1$ & $3: 1$ \\
\hline Contact angle (degrees) & $53.6 \pm 2.2$ & $52.3 \pm 3.1$ & $67.4 \pm 1.9$ \\
\hline
\end{tabular}

\section{Cell Spreading and Aggregation}

SEM observation showed that the hMSCs spread on the membranes prepared at chitosan:DNA ratios of 1:1 and 3:1. 
In contrast, a higher DNA content (chitosan:DNA $=1: 3$ ) suppressed cell spreading and caused cell aggregation (Fig. 2).

a

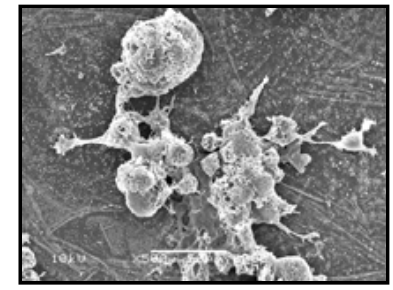

b

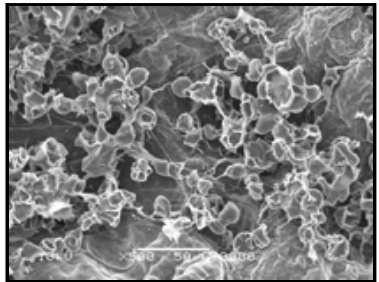

C

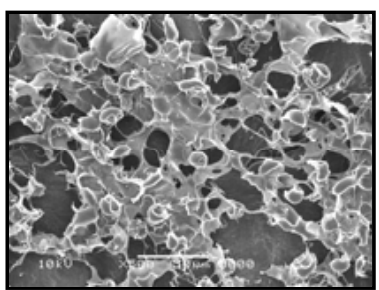

Fig. (2). SEM photographs of the chitosan/DNA polyelectrolyte complex membranes seeded with mesenchymal stem cells. The ratios of chitosan and DNA are (a) 1:3, (b) 1:1, and (c) 3:1.

\section{Cell Viability}

A WST-1 assay was used to assess the viability of the mesenchymal stem cells after $3 \mathrm{~h}$ culture on the polyelectrolyte complex membranes. Cell viability was higher on the membranes prepared at chitosan/DNA ratios of 1:1 and 3:1 than that on the membrane prepared at a chitosan/DNA ratio of 1:3 (Fig. 3).

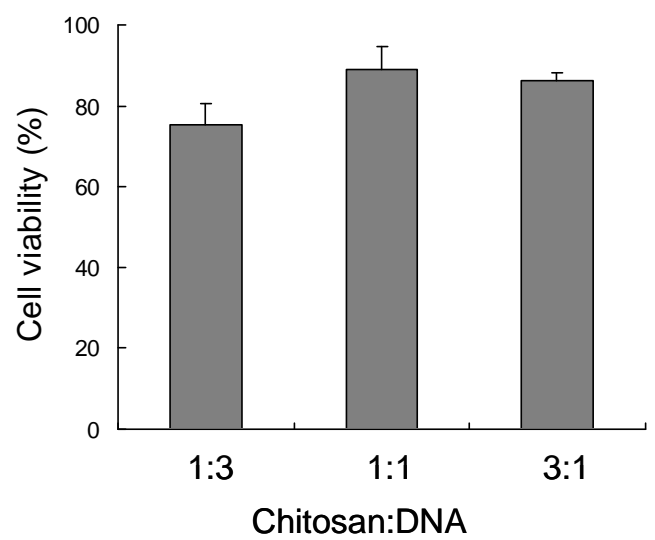

Fig. (3). Cell viabilities of the mesenchymal stem cells on the chitosan/DNA polyelectrolyte complex membranes with different ratio of chitosan and DNA. The values were normalized to $100 \%$ using that for cells on a polystyrene culture well plate.

\section{DISCUSSION}

The objective of the present study was to learn the true nature of the relationship between material surface properties and cell spreading/aggregation on chitosan/DNA polyelectrolyte complex membranes. Chitosan/DNA complex membranes having different surface properties were prepared by controlling the ratio of chitosan and DNA from 1:3 to $3: 1$. The membrane prepared at a chitosan/DNA ratio of $1: 3$ should have a negative charge because of the excess DNA. The membranes prepared at chitosan/DNA ratios of 1:1 and 3:1 should have a neutral and positive charge, respectively. The membrane surface was less hydrophilic when the ratio of chitosan was high.

Mesenchymal stem cells adhered and spread on the complex membranes prepared at chitosan/DNA ratios of 3:1 and $1: 1$. However, the cells on the membrane prepared at the chitosan/DNA ratio of 1:3 did not spread and aggregated to form spheroids. Cell viability on membranes prepared at chitosan/DNA ratios of 3:1 and 1:1 was higher than that on the 1:3 ratio membranes. These results suggest that the excess DNA in the complex suppressed cell spreading and viability. The negative charge of the DNA might be the reason for the inhibited cell spreading and low cell viability.

Chitosan/DNA polyelectrolyte complexes can be applied as a surface coating in addition to their use as a bulk material. However, their use as a surface coating is limited to hydrophilic surfaces.

Our study showed that a chitosan/DNA polyelectrolyte complex formed on treated polystyrene, but the membrane on the untreated plate broke during drying. The surface property of the PS substrate was found to affect the formation and shape stabilization of the polyelectrolyte complex membrane. The hydrophilicity of the treated polystyrene plate might stabilize the membrane via hydrogen bonding. Hydrophobic materials should be coated with a chitosan/DNA complex only after hydrophilizing the surface.

Finally, the study presented here demonstrated that chitosan/DNA polyelectrolyte complexes are useful for controlling cell spreading and aggregation.

\section{CONCLUSIONS}

Chitosan/DNA polyelectrolyte complex membranes with different ratios of DNA and chitosan were prepared by adjusting the ratio of the mixture from 3:1 to 1:3. The ratio of DNA and chitosan showed some effect on cell adhesion, spreading, and viability. The chitosan component facilitated cell adhesion and spreading, and maintained high cell viability, while the DNA component suppressed these properties.

\section{REFERENCES}

[1] Kumar MNVR, Muzzarelli RAA, Muzzarelli C, et al. Chitosan Chemistry and Pharmaceutical Perspectives. Chem Rev 2004; 104 : 6017-84.

[2] Muzzarelli RAA, Muzzarelli C. Chitosan Chemistry: Relevance to the Biomedical Sciences. Adv Polym Sci 2005; 186: 151-209.

[3] II'ina AV, Varlamov VP. Chitosan-based polyelectrolyte complexes: A review. Appl Biochem Microbiol 2005; 41: 5-11.

[4] Yi HM, Wu LQ, Bentley WE, et al. Biofabrication with chitosan. Biomacromolecules 2005; 6: 2881-94.

[5] Leong KW, Mao HQ, Truong-Le VL, et al. DNA-polycation nanospheres as non-viral gene delivery vehicles. J Control Release 1998; 53: 183-93.

[6] Erbacher P, Zou SM, Bettinger T, et al. Chitosan-based vector/DNA complexes for gene delivery: Biophysical characteristics and transfection ability. Pharmaceut Res 1998; 15: 1332-9. 
[7] Sato T, Ishii T, Okahata Y. In vitro gene delivery mediated by chitosan. Effect of pH, serum, and molecular mass of chitosan on the transfection efficiency. Biomaterials 2001; 22: 2075-80.

[8] Liu W, Sun S, Cao Z, et al. An investigation on the physicochemical properties of chitosan/DNA polyelectrolyte complexes. Biomaterials 2005; 26: 2705-11.

[9] Fukushima T, Hayakawa T, Kawaguchi M, et al. PBS buffer solutions with different $\mathrm{pH}$ values can change porosity of Chitosan/DNA complexes. Dent Mater J 2005; 24: 414-21.

[10] Fukushima T, Hayakawa T, Okamura K, et al. Buffer solution can control the porosity of Chitosan/DNA complexes. J Biomed Mater Res 2006; 76B: 121-9.

[11] Elbert DL, Hubbell JA. Reduction of fibrous adhesion formation by a copolymer possessing an affinity for anionic surfaces. J Biomed Mater Res 1998; 42: 55-65.
[12] Qiu Q, Sayer M, Kawaja M, et al. Attachment, morphology, and protein expression of rat marrow stromal cells cultured on charged substrate surfaces. J Biomed Mater Res 1998; 42: 117-27.

[13] Webb K, Hlady V, Tresco PA. Relative importance of surface wettability and charged functional groups on NIH 3T3 fibroblast attachment, spreading, and cytoskeletal organization. J Biomed Mater Res 1998; 41: 422-30.

[14] Anselme K, Bigerelle M, Noel B, et al. Qualitative and quantitative study of human osteoblast adhesion on materials with various surface roughnesses. J Biomed Mater Res 2000; 49: 155-66.

[15] Lo CM, Wang HB, Dembo M, et al. Cell movement is guided by the rigidity of the substrate. Biophys J 2000; 79: 144-52.

[16] Li B, Ma YX, Wang S, et al. Influence of carboxyl group density on neuron cell attachment and differentiation behavior: Gradientguided neurite outgrowth. Biomaterials 2005; 26: 4956-63. 